\title{
Sub-arcsecond resolution radio continuum observations of IRAS 20126+4104^
}

\author{
P. Hofner ${ }^{1,2}$, R. Cesaroni ${ }^{3}$, L. Olmi ${ }^{3,4}$, L. F. Rodríguez ${ }^{5}$, J. Martí ${ }^{6}$, and E. Araya ${ }^{1,2}$ \\ 1 Physics Department, New Mexico Tech, 801 Leroy Place, Socorro, NM 87801, USA \\ e-mail: phofner@nrao.edu \\ 2 National Radio Astronomy Observatory, PO Box O, Socorro, NM 87801, USA \\ 3 INAF - Osservatorio Astrofisico di Arcetri, Largo E. Fermi 5, 50125 Firenze, Italy \\ 4 Physics Department, University of Puerto Rico at Rio Piedras, San Juan, Puerto Rico 00931, USA \\ 5 Centro de Radioastronomía y Astrofísica, UNAM, Apdo. Postal 3-72, 58089 Morelia, Michoacan, México \\ 6 Departamento de Física, Escuela Politécnica Superior, Universidad de Jaén, Calle Virgen de la Cabeza 2, 23071 Jaén, Spain
}

Received 7 June 2006 / Accepted 27 October 2006

\section{ABSTRACT}

\begin{abstract}
Context. The detailed physical processes that lead to the formation of massive stars are still unknown. Observations that probe linear scales as small as $100 \mathrm{AU}$ are necessary for improving our understanding in this area.

Aims. We present high angular-resolution and high-sensitivity multi-frequency radio observations of the deeply embedded massive protostar located in the IRAS 20126+4104 core region, with the aim of investigating the nature of the radio continuum emission from the deeply embedded massive protostar.

Methods. The observations were performed with the Very Large Array in several continuum bands with wavelengths between 20 and $0.7 \mathrm{~cm}$ in the $\mathrm{A}$ and/or B configurations.

Results. At $3.6 \mathrm{~cm}$ we resolve the emission of the IRAS 20126+4104 core into 3 components. The emission from the two northern sources is consistent with free-free emission from ionized gas with a density gradient. Most likely the ionization is caused by UV radiation from the cooling region of a shock; i.e. the ionization is caused by the jet driven by the IRAS $20126+4104$ protostar. The morphology and measured flux densities of the southern source is consistent with emission from an optically thin jet, most likely also due to shock ionization. A simple radiative transfer model shows that the spectral energy distribution of IRAS $20126+4104$ is consistent with an accretion disk embedded in a spherical halo. We also report the discovery of a highly variable radio source near the IRAS 20126+4104 core, which is most likely gyrosynchotron emission from a low-mass pre-main sequence star.
\end{abstract}

Key words. stars: formation - ISM: jets and outflows - ISM: individual objects: IRAS 20126+4104

\section{Introduction}

The luminous $\left(L \approx 10^{4} L_{\odot}\right)$ source IRAS $20126+4104$ is one of the best candidates for a young massive object in a very early stage of evolution. Due to its relatively unconfused structure and near distance $(1.7 \mathrm{kpc})$, it has recently been studied by several authors. Interferometric observations of many different molecular probes, as well as mm-continuum, established the existence of a disk-outflow system in the IRAS $20126+4104$ core region. On angular scales $>30^{\prime \prime}$ the outflow is oriented $\mathrm{N}-\mathrm{S}$ (Shepherd et al. 2000), but the orientation is SE-NW on smaller scales (Cesaroni et al. 1997, 1999, hereafter C97 and C99, respectively). The change in outflow direction is possibly due to the precesssion of the flow axis caused by a nearby companion (Shepherd et al. 2000). On the other hand, observations of dense gas tracers have found the central molecular gas to be oriented perpendicular to the flow axis, with a velocity gradient directed NE-SW. This configuration is interpreted as a molecular disk around the central object that is powering the flow (Zhang et al. 1998; C97, C99). The kinematics of the disk was

* Based on data collected with the NRAO Very Large Array. The National Radio Astronomy Observatory is a facility of the National Science Foundation operated under cooperative agreement by Associated Universities, Inc. recently studied in detail by Cesaroni et al. (2005, hereafter C05) using interferometric observations of the $\mathrm{C}^{34} \mathrm{~S}(2-1)$ and (5-4) lines. These authors confirmed that the velocity field is nearly Keplerian and derived a mass of the central object of about $7 M_{\odot}$. The molecular line data also suggest that the central object is still in a phase of mass accretion, with a rate of approximately $10^{-3} M_{\odot}$ year $^{-1}$.

The molecular line and dust continuum observations described above had angular resolution between 0.'6-3", i.e. probing linear scales of 1000-5000 AU, and we decided to study the interior region close to the protostar using VLA radio continuum observations. Our earlier B-configuration $3.6 \mathrm{~cm}$ map (Hofner et al. 1999) showed two sources, both elongated in the direction of the massive flow, which we tentatively interpreted as a double system of ionized jets at the base of the molecular flow in IRAS $20126+4104$

The exact position of the massive proto-star and the nature of the continuum emission in the IRAS $20126+4104$ core are still uncertain. Hofner et al. (1999) assumed the massive proto-star to reside between the two northern $3.6 \mathrm{~cm}$ maxima, so that the ionized jet would be bipolar. The mm observations that identified the flow and disk components (C97, C99, C05) have astrometric precisions of about $0{ }^{\prime} 9$, i.e. not sufficient to determine whether 
this assumption is correct. Trinidad et al. (2005) observed the $\mathrm{H}_{2} \mathrm{O}$ masers in IRAS20126 + 4104 with the VLA and found a rotational component, which could be interpreted as Keplerian rotation if the central protostar were located to the NW of the $\mathrm{cm}$ continuum peak. Moscadelli et al. (2005) performed VLBI observations of the $22 \mathrm{GHz} \mathrm{H_{2 }} \mathrm{O}$ masers in IRAS $20126+4104$ and detected proper motions along the jet axis with radial expansion speeds between $34-112 \mathrm{~km} \mathrm{~s}^{-1}$. Thus their data clearly indicate that the $\mathrm{H}_{2} \mathrm{O}$ masers are part of the flow/jet. The rotational component found by Trinidad et al. (2005) could then be explained by rotation of the jet, which is an important prediction of magnetized disk wind models (e.g. Pudritz \& Banerjee 2005), and has already been observed in low-mass young stellar objects (Woitas et al. 2005; Coffey et al. 2004).

Moscadelli et al. $(2000,2005)$ present a model where $\mathrm{H}_{2} \mathrm{O}$ masers arise from the surface of a conical jet, with the position of the driving object located near the $3.6 \mathrm{~cm}$ peak. In an additional recent study, Edris et al. (2005) observed the $1665 \mathrm{MHz}$ maser lines of $\mathrm{OH}$ and the $6.7 \mathrm{GHz}$ maser lines of $\mathrm{CH}_{3} \mathrm{OH}$ toward IRAS $20126+4104$ with MERLIN. The angular resolution and positional uncertainty were 0.15 and $0 . ' 025$ for $\mathrm{OH}$, and $0 .{ }^{\prime} 025$ and $0 .{ }^{\prime} 012$ for $\mathrm{CH}_{3} \mathrm{OH}$. Similar to previous observations of other dense molecular gas tracers in IRAS 20126+4104 (see above), their data showed an elongated structure of size 1".2 (2000 AU) with a NE-SW velocity gradient, consistent with Keplerian rotation around a central object of mass 5-20 $M_{\odot}$. However, the hypothetical center of this disk is located 0.' 3 to the $\mathrm{SE}$ of the strongest $\mathrm{cm}$ continuum component. Additional information supporting the hypothesis that the central protostar might be located between the strongest $\mathrm{OH}$ emission peaks SE of the $3.6 \mathrm{~cm}$ continuum source comes from the NIR data of Sridharan et al. (2005). With an absolute positional uncertainty of $0{ }^{\prime} 15$, these authors imaged a dark extinction lane (see their Fig. 2), which presumably traces the circumstellar disk around the massive protostar in IRAS $20126+4104$. The strongest $\mathrm{OH}$ emission is located at the two ends of the dark lane (Edris et al. 2005), consistent with the circumstellar disk interpretation.

In this paper we present multi-frequency VLA radio continuum data with subarcsecond angular resolution and sub-mJy sensitivity, to further study the environment very near the massive protostar. Section 2 describes details of the observations and data reduction, and in Sect. 3 we present the basic observational results. We discuss these findings in Sect. 4, and conclude with a summary in Sect. 5.

\section{Observations and data reduction}

We observed IRAS 20126+4104 with the VLA in standard continuum mode at wavelengths of $0.7,1.3,3.6,6$, and $20 \mathrm{~cm}$ on several dates between 2000-2002, and also obtained $2 \mathrm{~cm}$ continuum data from the VLA archive. Details of these observations are given below.

\subsection{The $0.7 \mathrm{~cm}$ and $1.3 \mathrm{~cm}$ observations}

\subsubsection{B-configuration}

The observations were carried out in three 8-hour sessions on January 2, 7, and 8, 2000, with the VLA in the B-configuration. We used the fast switching technique which consists of rapidly alternating observations of IRAS 20126+4104 and the nearby calibrator $\mathrm{J} 2007+404$ with total cycle times of 130 and $80 \mathrm{~s}$ at wavelengths of 1.3 and $0.7 \mathrm{~cm}$, respectively. Pointing corrections were determined hourly and applied on-line. The phase-center was $\alpha(J 2000)=20^{\mathrm{h}} 14^{\mathrm{m}} 26^{\mathrm{s}} .036, \delta(J 2000)=$ $41^{\circ} 13^{\prime} 32^{\prime \prime}$.52. Flux density was calibrated by observations of $3 \mathrm{C} 84$ for which we assumed values of 15.06 and $7.69 \mathrm{Jy}$ at 1.3 and $0.7 \mathrm{~cm}$, respectively. On each day we observed for about $6 \mathrm{~h}$ at $0.7 \mathrm{~cm}$, switching to $1.3 \mathrm{~cm}$ during two hours near the transit of IRAS 20126+4104. The data of January 2, 2000 were affected by rapid atmospheric phase variations that led to substantially poorer image quality and we did not use data taken on that day. The data of January 7 and 8 were separately edited, calibrated, and Fourier-transformed using the AIPS software package. Both days gave consistent results and the UV data were subsequently merged for each wavelength.

\subsubsection{A-configuration}

The A-configuration observations were carried out in two 8-h sessions on February 28 and March 02, 2002 in a fashion similar to the B-configuration observations described above. The cycle time used for fast switching was $80 \mathrm{~s}$ at both wavelengths. As in the B-configuration observations, we used J2007+404 as the secondary calibrator. Flux calibration was achieved by observations of 3C286, in conjunction with a source model provided by NRAO. The data taken on the two different days were separately edited and calibrated, and images from the two datasets were compared and found consistent. The UV data were subsequently combined for each wavelength. Images were made for the combined datasets at each wavelength. Judging from the variation in the flux density of the bright unresolved source in the IRAS $20126+4104$ core (N1, see below) measured on different days and in different configurations, we estimate that our flux calibration is better than $20 \%$ at both $0.7 \mathrm{~cm}$ and $1.3 \mathrm{~cm}$.

We also combined the B- and A-configuration data sets and made images at both wavelengths. The final uniformly weighted $1.3 \mathrm{~cm}$ image has a synthesized beam of $0 .^{\prime} 087 \times 0 .{ }^{\prime} 073, \mathrm{PA}=$ $17.7^{\circ}$, and $\mathrm{rms}$ noise of $51 \mu \mathrm{Jy}$ beam $^{-1}$. The final uniformlyweighted $0.7 \mathrm{~cm}$ image has a synthesized beam of $0 .{ }^{\prime} 047 \times 0 .{ }^{\prime} 041$, $\mathrm{PA}=-52.0^{\circ}$, and $\mathrm{rms}$ noise of $76 \mu \mathrm{Jy} \mathrm{beam}^{-1}$.

\subsection{The $2.0 \mathrm{~cm}$ archival data}

We searched the VLA archive for past observations of IRAS 20126+4104 and include here a $2.0 \mathrm{~cm}$ measurement taken in December 1999 when the VLA was in the Bconfiguration. As in our own observations, B2005+403 (same as J2007+404 in B1950 coordinates) was used to calibrate phase and amplitude variations. The reduction procedure was similar to what is decribed above; however, no measurement of a flux calibrator source was available for these data. We set the flux density scale by assuming a flux of $1.95 \mathrm{Jy}$ for B2005+403 based on the average of measurements of this source during December 1999March 2000. The scatter in these measurements during this time is about $15 \%$. Due to the long calibrator-source cycle (typically 20 minutes), residual phase errors result in relatively poor map quality, and we conservatively assume an error on the flux measurement of $30 \%$. The final naturally weighted $2.0 \mathrm{~cm}$ image has a synthesized beam of $0.59 \times 0.46, \mathrm{PA}=-56.2^{\circ}$, and rms noise of $65.2 \mu \mathrm{Jy}_{\text {beam }}$ $^{-1}$.

\subsection{The $3.6 \mathrm{~cm}$ observations}

We observed IRAS $20126+4104$ at a wavelength of $3.6 \mathrm{~cm}$ on November 26, 2000 with the VLA in the A-configuration for a total time of 6 hours. We alternated 12 min scans on 
IRAS $20126+4104$ with 2 min scans on the nearby calibrator B2005+403. Flux calibration was derived from observations of $3 \mathrm{C} 84$ for which we assumed a flux density of $3.15 \mathrm{Jy}$ at $3.6 \mathrm{~cm}$. The phase center was $\alpha(B 1950)=20^{\mathrm{h}} 12^{\mathrm{m}} 41^{\mathrm{s}} .000$, $\delta(B 1950)=41^{\circ} 04^{\prime} 25^{\prime \prime}$. 00 . The data were edited, calibrated, and Fourier-transformed using the AIPS software package.

We compared an image made from the A-configuration data with our earlier $3.6 \mathrm{~cm}$ image obtained from B-configuration data (Hofner et al. 1999). The two naturally weighted maps agree within $10 \%$ in integrated flux, and the observed morphology of the A-configuration map is consistent with the B-configuration data. Furthermore, we made an image of the A-configuration data restricting the $\mathrm{UV}$ range to that of the B-configuration data and subsequently subtracted this map from the B-configuration image. The resulting difference map showed no significant emission. We subsequently merged A- and B-configuration data in the UV domain and produced images using AIPS task IMAGR with a variety of weighting schemes. Our final natural-weighted map has a synthesized beam of $0 .{ }^{\prime} 27 \times 0.26, \mathrm{PA}=-14.3^{\circ}$, and rms noise of $9.0 \mu \mathrm{Jy}_{\text {beam }^{-1}}$.

\subsection{The $6 \mathrm{~cm}$ and $20 \mathrm{~cm}$ observations}

Short integrations toward IRAS $20126+4104$ at wavelengths of 6 and $20 \mathrm{~cm}$ were taken on May 3, 2002 with the VLA in the A-configuration. No sources were detected at either wavelength with an rms value of $0.05 \mathrm{mJy} \mathrm{beam}^{-1}$ at $20 \mathrm{~cm}$, and $0.1 \mathrm{mJy}^{\text {beam }}{ }^{-1}$ at $6 \mathrm{~cm}$.

\section{Results}

\subsection{Morphology}

In Fig. 1, top panel, we show a contour plot of a ROBUST 0 weighted $3.6 \mathrm{~cm}$ map. As expected from our previous B-configuration observations (Hofner et al. 1999), several components are detected at $3.6 \mathrm{~cm}$. As indicated in the figure, we refer to these as N1, N2, and S, respectively. For the northern source our previous $3.6 \mathrm{~cm}$ B-configuration observations (Hofner et al. 1999) showed an elongated structure, which we tentatively interpreted as an ionized jet. With the higher angular resolution of the A-configuration data, we find that the northern source breaks up into two distinct sources. Source N2 is located approximately 0.'5 toward the NW from N1, and most of its emission arises from an unresolved structure. The position angle between the two sources is $112^{\circ}$, near the position angle of the large-scale flow measured by C97. In the bottom panel of Fig. 1 we show a $3.6 \mathrm{~cm}$ contour map made with increased angular resolution by downweighting the shorter baselines (ROBUST -1). Due to weighting noise the significance of the observed features is lower compared to the ROBUST 0 map shown in the upper panel; however, the increased angular resolution provides new information. In particular, the morphology of $\mathrm{N} 2$ is suggestive of a bow shock. In this figure we also show the positions of water masers from the studies of Tofani et al. (1995), Moscadelli et al. (2000, 2005), and Trinidad et al. (2005), as well as the outflow direction obtained from the conical flow model of Moscadelli et al. (2005). Most water maser are located in front of N1, but some water masers are associated with N2. Also, the data suggests that the southern source might be breaking up into individual components.

In contrast to the northern sources, our $3.6 \mathrm{~cm}$ data resolve the southern source into several synthesized beams along its major axis. Its elongated structure is well-fit by a 2-D Gaussian of

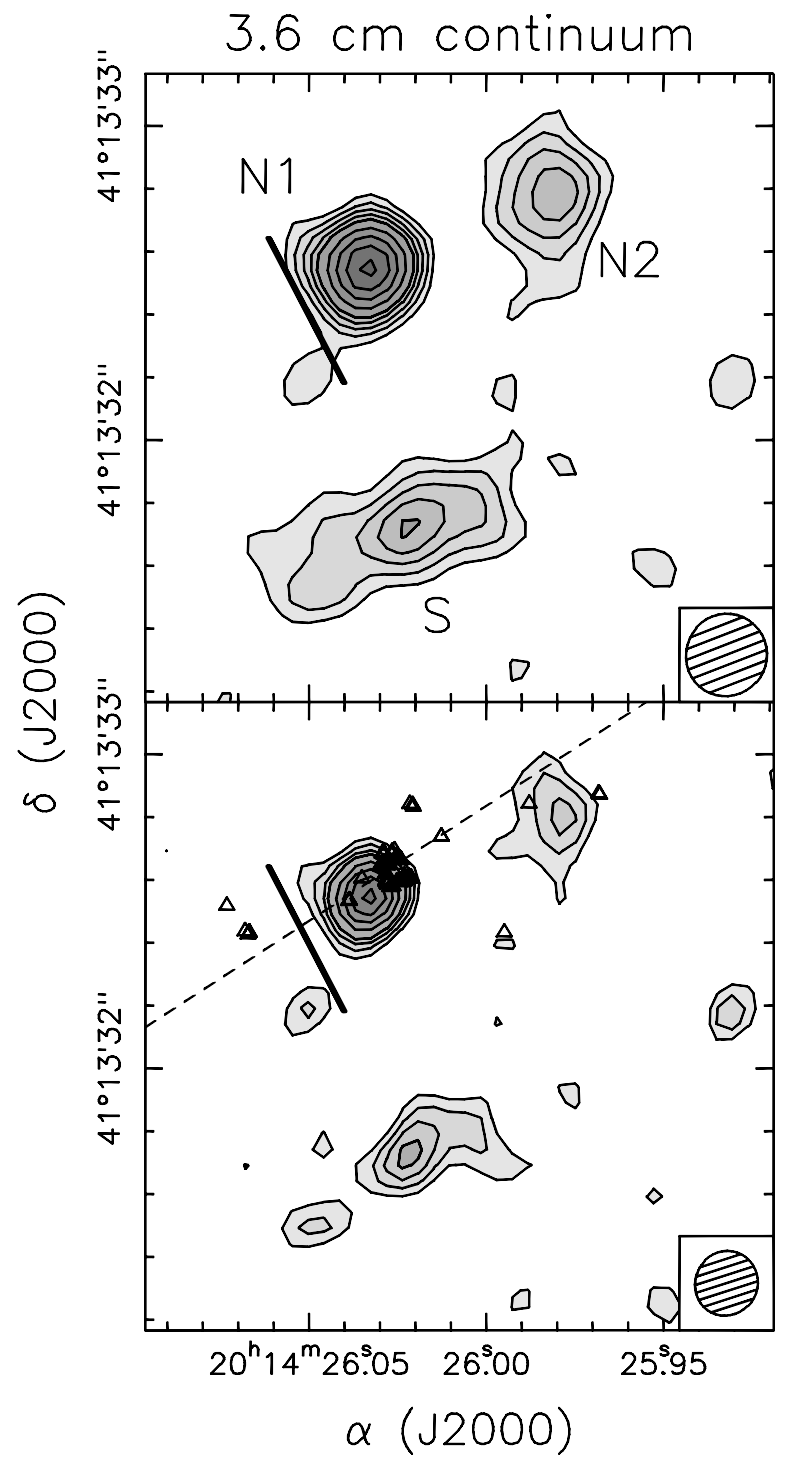

Fig. 1. Contour plots of the $3.6 \mathrm{~cm}$ continuum emission toward IRAS 20126+4104. In the top panel we show a ROBUST 0 weighted map. Contour levels are 0.017 to $0.057 \mathrm{mJy} \mathrm{beam}^{-1}$ in

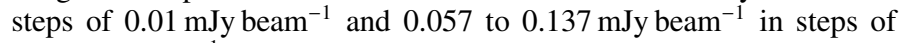
$0.02 \mathrm{mJy}$ beam $^{-1}$. The three continuum components are labeled as N1, $\mathrm{N} 2$, and $\mathrm{S}$. The synthesized beam is shown in the lower right corner. The thick line to the SE of N1 shows the position of the dark lane reported by Edris et al. (2005). The bottom panel shows our $3.6 \mathrm{~cm}$ ROBUST -1 map. Contour levels are 0.02 to $0.05 \mathrm{mJy}^{-1}$ beam $^{-1}$ in steps of $0.01 \mathrm{mJy} \mathrm{beam}^{-1}$ and 0.05 to $0.13 \mathrm{mJy}$ beam $^{-1}$ in steps of $0.02 \mathrm{mJy}$ beam $^{-1}$. Here we also show the position of water masers (Tofani et al. 1995; Moscadelli et al. 2000, 2005; Trinidad et al. 2005) as triangles, as well as their outflow direction (dashed line, Moscadelli et al. 2005)

FWHM 0.'62 $\times 0$. ' $18(1054 \times 306 \mathrm{AU})$ along a position angle of $107^{\circ}$. The smooth elongated appearance of this source is highly suggestive of an ionized jet. As found for the northern jet, the orientation of this source is also near the position angle of the large-scale molecular flow.

Due to higher receiver noise the 1.3 and $0.7 \mathrm{~cm}$ maps have rms noise levels of 5 and 8 times that of the $3.6 \mathrm{~cm}$ map, and only N1 is detected at these wavelengths (Fig. 2). We determined the peak positions and sizes of the detected sources at each wavelength by 2-D Gaussian fits, and extracted the total flux by integrating over an area just covering each source. For the 


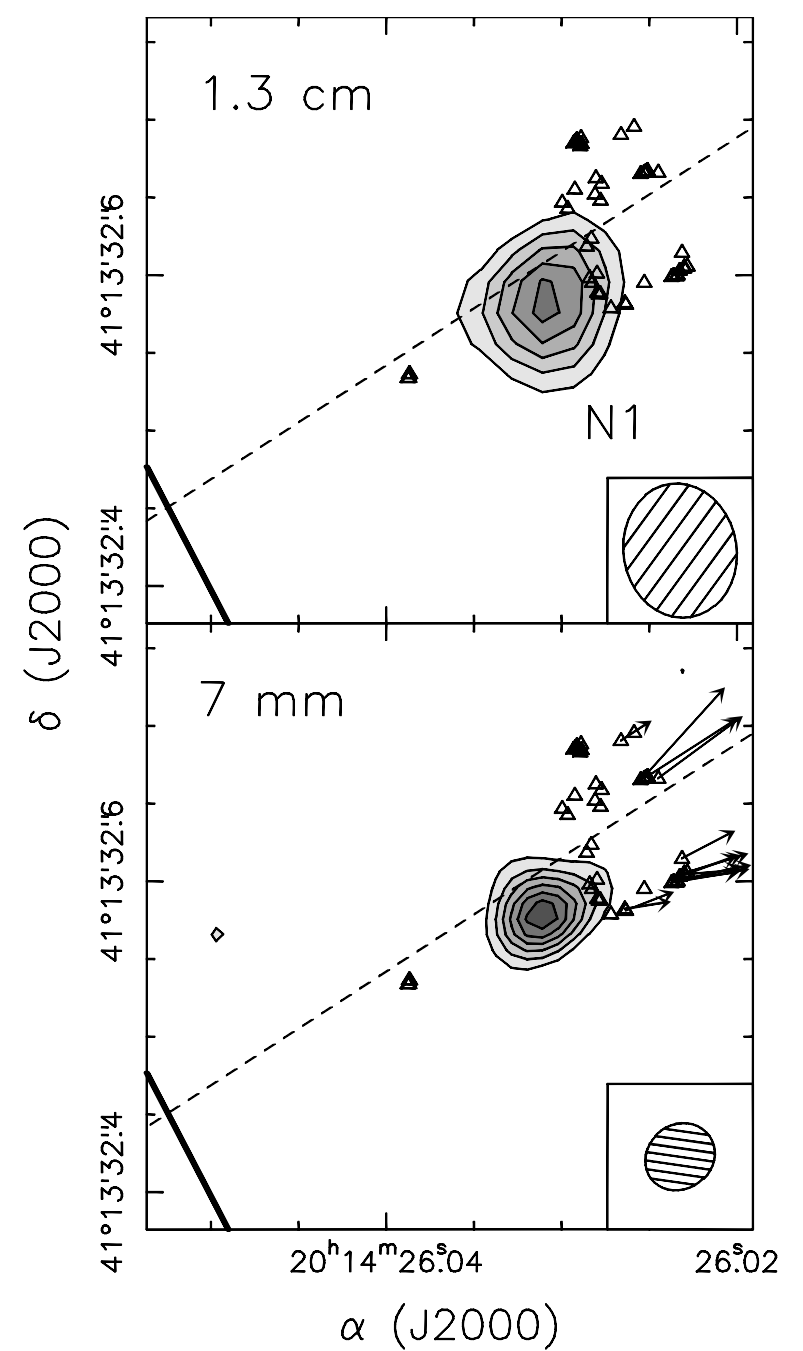

Fig. 2. Top: $1.3 \mathrm{~cm}$ continuum emission toward IRAS 20126+4104. Contour levels are from 0.15 to $0.55 \mathrm{mJy}^{-1} \mathrm{beam}^{-1}$ in steps of $0.1 \mathrm{mJy}$ beam $^{-1}$. The synthesized beam is shown in the lower right corner. The thick black line to the SE of N1 shows the position of the dark lane reported by Edris et al. (2005). The positions of water masers are shown as triangles, and their flow direction is shown as a dashed line. The bottom panel shows our $7 \mathrm{~mm}$ map. Contour levels are from 0.22 to $1.22 \mathrm{mJy}_{\text {beam }}{ }^{-1}$ in steps of $0.2 \mathrm{mJy}_{\text {beam }}{ }^{-1}$. Here we also show the proper motion of individual water masers measured by Moscadelli et al. (2005) (arrows).

non-detected sources we also give $3 \sigma$ limits on the flux density and the corresponding limit on the brightness temperature within a synthesized beam. These data are presented in Table 1.

The measured positions of $\mathrm{N} 1$ at $3.6,1.3$, and $0.7 \mathrm{~cm}$ are identical within our astrometric accuracy. They also agree with the recent $1.3 \mathrm{~cm}$ measurement of Trinidad et al. (2005) to within 0 .'02. The larger positional offset of N1 obtained from the $2 \mathrm{~cm}$ map can be explained by the relatively low map quality caused by poor phase calibration in conjunction with the large synthesized beam. The position of N1 varied by at most 0. ' 05 between any image taken in this observational campaign, and therefore it appears that N1 is not coincident with the putative position of the massive proto-star as indicated by the $\mathrm{OH}$ observations of Edris et al. (2005) and the dark lane detected in the NIR observations of Sridharan et al. (2005), but offset by about $0 . ' 3$ to the NW (see Figs. 1 and 2).
$\mathrm{N} 1$ is essentially unresolved at 1.3 and $3.6 \mathrm{~cm}$ with upper limits on the size of 0 ' $^{\prime} 05$ and 0 .' $^{\prime} 13$, corresponding to linear sizes of 85 and $221 \mathrm{AU}$, respectively. In Fig. 2 we show our ROBUST -1 weighted 1.3 and $0.7 \mathrm{~cm}$ maps (upper and lower panels, respectively). At a wavelength of $0.7 \mathrm{~cm}, \mathrm{~N} 1$ is marginally resolved along a position angle of $125^{\circ}$, with a deconvolved FWHM along that direction of $0{ }^{\prime} 02$, corresponding to a linear size of $34 \mathrm{AU}$.

\subsection{A variable radio source}

We report the discovery of a highly variable radio source near the IRAS $20126+4104$ core region. In the 12 observations reported in this paper we detected this source 6 times, and it was below our detection limits for 6 times. A summary of the fluxes is given in Table 2, and we show the position of this source in Fig. 3. The variable radio source (hereafter I20var) is located about $5^{\prime \prime}$ to the SE of IRAS $20126+4104 \mathrm{~N} 1$ at position $\alpha(J 2000)=20^{\mathrm{h}} 14^{\mathrm{m}} 26.238, \delta(J 2000)=41^{\circ} 13^{\prime} 28^{\prime \prime} .07$. The measured source position varied by less than $0 .^{\prime} 01$ for the 6 detections reported in this paper. By inspection of Table 2 it is clear that I20var undergoes strong variation in its radio flux. The strongest variation is seen at $3.6 \mathrm{~cm}$ where the flux changed by a factor of more than 40 between 1998 and 2000. The $K$ and $Q$-band observations of 2000 were obtained on subsequent days and the flux changed by factors of 5 and $>3$ at these wavelengths. The $K$ - and $Q$-band observations of 2002 were separated by 3 days and the measured flux variation was $>19$ and $>15$, respectively. The observations described above had typical lengths of a few hours, and we were not able to detect any change in flux during the observations. We conclude that I20var shows variablity in flux of a factor 20 or larger within a time period of days.

The nature of the radio emission from I20var is difficult to ascertain because we detected the source only on two occasions at more than one wavelength, and the shape of the radio spectrum is unknown. The spectral index between 1.3 and $0.7 \mathrm{~cm}$ was $-0.5 \pm 0.2$ on January 7,2000 , and $0.3 \pm 0.2$ on March 2, 2002, which marginally indicates a change in the spectral slope. I20var remains unresolved even in the ROBUST -1 weighted map of our A-configuration $0.7 \mathrm{~cm}$ data with a synthesized beam of 0 ' $^{\prime} 042 \times 00^{\prime} 037$. Adopting a limit on the source size of half the synthesized beam implies a lower limit on the brightness temperature of $T_{\mathrm{B}}>7400 \mathrm{~K}$.

We found no extragalactic counterpart for I20var in the NED database. This is consistent with the probability of detecting of an extragalactic radio source at a given flux level: at $3.6 \mathrm{~cm}$, the probability of detecting an $S_{v}>0.06 \mathrm{mJy}$ extragalactic source in a $30^{\prime \prime} \times 30^{\prime \prime}$ box is $\sim 4 \%$ (see Gómez et al. 2002; Windhorst et al. 1993). Hence, we consider an extragalactic origin of the variable source unlikely.

An alternative explanation is variable radio emission from a pre-main sequence star. For example, the binary system $\theta^{1}$ Ori A composed of a massive star and a T Tauri star is a well-known variable radio source (Felli et al. 1993). The variability is possibly caused by non-thermal flaring activity on the surface of the $\mathrm{T}$ Tauri companion. This source shows a factor of $\sim 45$ in variability at $\mathrm{cm}$ wavelengths, from $\sim 2$ to $\sim 90 \mathrm{mJy}$ on timescales of days (10 to 20 days), with no perceptible variation on short timescales (on the order of minutes), and a variability in the spectral index between approximately -0.5 and +0.7 (Felli et al. 1993). These characteristics are remarkably similar to those derived from our observations of I20var. Furthermore, if $\theta^{1}$ Ori A were located at the distance of IRAS 20126+4104, then its flux 
Table 1. Observed continuum parameters.

\begin{tabular}{ccccccr}
\hline \hline Source & $\begin{array}{c}\text { Wavelength } \\
(\mathrm{cm})\end{array}$ & $\begin{array}{c}\alpha(\mathrm{J} 2000) \\
(\mathrm{h} \mathrm{m} \mathrm{s})\end{array}$ & $\begin{array}{c}\delta(\mathrm{J} 2000) \\
\left({ }^{\circ}{ }^{\prime}{ }^{\prime \prime}\right)\end{array}$ & $\begin{array}{c}S_{v} \\
(\mathrm{mJy})\end{array}$ & $\begin{array}{c}\text { Size } \\
\left({ }^{\prime \prime}\right)\end{array}$ & $\begin{array}{r}T_{\mathrm{B}} \\
(\mathrm{K})\end{array}$ \\
\hline $\mathrm{N} 1$ & 3.6 & 201426.036 & 411332.54 & 0.15 & $<0.09$ & $>316$ \\
& 2.0 & 201426.054 & 411332.59 & 0.39 & $<0.25$ & $>34$ \\
& 1.3 & 201426.031 & 411332.58 & 0.56 & $<0.04$ & $>847$ \\
$\mathrm{~N} 2$ & 0.7 & 201426.031 & 411332.58 & 1.38 & $0.02 \times<0.02,122^{\circ}$ & $>2245$ \\
& 3.6 & 201425.984 & 411332.77 & 0.07 & $<0.09$ & $>150$ \\
& 1.3 & - & - & $<0.10^{a}$ & - & $<151^{b}$ \\
$\mathrm{~S}$ & 0.7 & - & - & $<0.19^{a}$ & - & $<309^{b}$ \\
& 3.6 & 201426.025 & 411331.71 & 0.15 & $0.70 \times 0.15,111^{\circ}$ & 25 \\
& 1.3 & - & - & $<0.10^{a}$ & - & $<151^{b}$ \\
& 0.7 & - & - & $<0.19^{a}$ & - & $<309^{b}$ \\
\hline
\end{tabular}

${ }^{a}$ Non-detection. Limits are 3 times rms noise value in map.

${ }^{b}$ Limiting brightness temperature assuming a source size equal to the synthesized beam.

Table 2. The variable source.

\begin{tabular}{lcr}
\hline \hline Date & $\begin{array}{c}\text { Wavelength } \\
(\mathrm{cm})\end{array}$ & $\begin{array}{r}S_{v} \\
(\mathrm{mJy})\end{array}$ \\
\hline 03-MAY-2002 & 20 & $<0.15^{a}$ \\
& & $<0.30^{a}$ \\
03-MAY-2002 & 6.0 & $0.04^{b}$ \\
& & 1.63 \\
18-AUG-1998 & 3.6 & \\
26-NOV-2000 & 3.6 & \\
& & \\
12-DEC-1999 & 2.0 & $<0.20^{a}$ \\
& & 1.55 \\
07-JAN-2000 & 1.3 & 0.28 \\
08-JAN-2000 & 1.3 & $<0.19^{a}$ \\
28-FEB-2002 & 1.3 & 3.66 \\
02-MAR-2002 & 1.3 & \\
& & 1.10 \\
07-JAN-2000 & 0.7 & $<0.37^{a}$ \\
08-JAN-2000 & 0.7 & 4.44 \\
28-FEB-2002 & 0.7 & $<0.29^{a}$ \\
02-MAR-2002 & 0.7 & \\
\hline
\end{tabular}

${ }^{a}$ Limits are 3 times rms noise value in map.

${ }^{b}$ From Hofner et al. (1999).

density would vary between a tenth of a mJy to a few mJy, which is the same range of variability observed in I20var. Another similarity between the radio-variable source $\theta^{1}$ Ori A and I20var is the lack of measurable circular polarization. For the main outburst of $\theta^{1}$ Ori A reported by Felli et al. (1993), they found no $\mathrm{V}$ polarization with an upper limit of $0.3 \%$, and in the case of the main $3.6 \mathrm{~cm}$ outburst of I20var (see Table 2), we found no V polarization within $2 \%$ of the I value.

Based on the 2MASS near-infrared maps toward the position of I20var, and other near infrared observations reported in the literature (C97; Ayala et al. 1998) we found no near-infrared counterpart of I20var up to $\sim 16$ th mag in the $2.2 \mu \mathrm{m}$ band. On the other hand, C99 detected extended $3 \mathrm{~mm}$ emission around the IRAS 20126+4104 core region, which encompasses the position of I20var. Assuming that the $3 \mathrm{~mm}$ continuum emission toward the position of I20var is due to thermal dust at a temperature of $100 \mathrm{~K}$, a dust opacity of $\kappa_{3 \mathrm{~mm}}=0.1 \mathrm{~cm}^{2} \mathrm{~g}^{-1}$, and a gas-to-dust ratio of 100 , then the column density toward the line of sight of I20var would be $N_{\mathrm{H}} \sim 10^{23} \mathrm{~cm}^{-2}$. This column density implies a visual extinction of roughly $A_{\mathrm{v}} \sim 50 \mathrm{mag}$, which can account for the non-detection of a T Tauri star in the near infrared (e.g.,

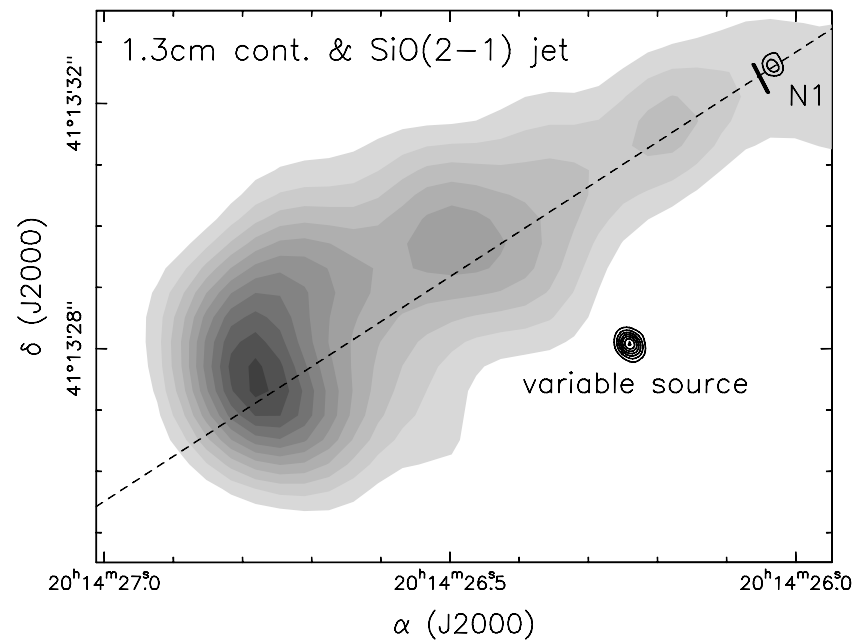

Fig. 3. Our $1.3 \mathrm{~cm}$ continuum map toward IRAS 20121+4104 taken on March 2, 2002 with the VLA A-configuration (contours), overlayed on a $\mathrm{SiO}(2-1)$ grey-scale plot from $\mathrm{C} 99.1 .3 \mathrm{~cm}$ contour levels are from 0.3 to $1.5 \mathrm{mJy}$ beam ${ }^{-1}$ in steps of $0.2 \mathrm{mJy}_{\text {beam }}{ }^{-1}$. The variable source was in a "high" state on this date, but was not present at very low noise levels just three days earlier. The grey scale $\mathrm{SiO}(2-1)$ emission illustrates the orientation of the molecular jet. As before, the dashed line marks the direction of the molecular flow and the perpendicular thick line the position of the dark lane (Edris et al. 2005).

Garmire et al. 2000). For example, out of $773.6 \mathrm{~cm}$ radio sources in Orion, only 49 have a near-infrared counterpart (Zapata et al. 2004). Thus, the non-detection of a near-infrared counterpart of a possible $T$ Tauri star located at a distance $\sim 4$ times that of Orion is not surprising.

In summary, although our data cannot establish the nature of I20var with certainty, we believe that gyrosynchrotron radiation from an active magnetosphere in a low-mass pre-main sequence star is the most likely cause of the radio emission from I20var.

\section{Discussion}

\subsection{IRAS $20126+4104 N 1$ and N2}

We now return to the continuum sources in the IRAS 20126+4104 core region. As discussed above, detection at multiple frequencies were only made for the strongest source N1. In Fig. 4 we show the radio-mm SED for this source, including VLA data (Hofner et al. 1999, and this work) and data 


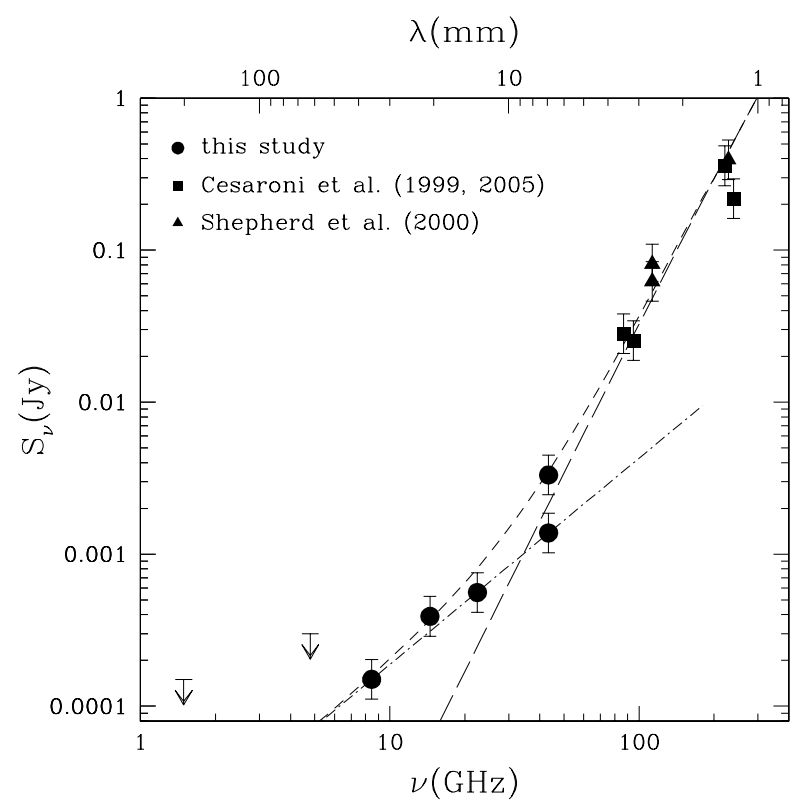

Fig. 4. Radio/mm spectral energy distribution of the IRAS 20126+4104 core region. Filled circles show the $\mathrm{cm}$ data points measured with the VLA, and filled squares and triangles show $\mathrm{mm}$ interferometric measurements. At $7 \mathrm{~mm}$ we also include the datapoint of Hofner et al. (1999) that was obtained with the VLA in the D-configuration (upper point). Arrows indicate the upper limits obtained for 6 and $20 \mathrm{~cm}$ in this work. The dot-dashed line shows a power law with slope 1.36, the long dashed line is the expected thermal emission from the accretion disk, and the short-dashed line shows the sum of the two spectral components.

obtained with mm-interferometers (C99; C05; Shepherd et al. 2000).

At $7 \mathrm{~mm}$ two datapoints are shown. The upper datapoint was taken with the VLA in the D-configuration (Hofner et al. 1999) with a beam of $1^{\prime \prime} .7 \times 11^{\prime \prime} 0$, and the lower datapoint is from this work, including both VLA B- and A-configuration data. The D-configuration point lies very close to what is predicted from optically thin dust emission based on the 3 and $1.3 \mathrm{~mm}$ data. C99 and C05 measured a deconvolved diameter of the compact dust disk of 0.64 , and derived a mass between 0.65-3.6 $M_{\odot}$ depending on the adopted value for the dust opacity. In our high-resolution data we only recover about $30 \%$ of the D-configuration $7 \mathrm{~mm}$ flux, which confirms that the dust component is quite extended with respect to the synthesized beam of the $\mathrm{A}+\mathrm{B}$ configuration data. If the dust component traces a disk of diameter $0 .{ }^{\prime} 64$ and thickness $0 .{ }^{\prime} 05$ and were smoothly distributed, the expected brightness at $7 \mathrm{~mm}$ would be $0.1 \mathrm{mJy}$ beam $^{-1}$, i.e. below our detection limit of the $\mathrm{A}+\mathrm{B}$ VLA configuration data. More compact dust distributions are also compatible with the flux loss; however in this case, the geometrical thickness of the disk must be larger than 0 .' $^{\prime} 05$.

In our A+B-configuration VLA data, we detect a total $7 \mathrm{~mm}$ flux of $1.38 \mathrm{mJy}$ for $\mathrm{N} 1$, and also begin to resolve the source along its major axis with a resulting lower limit on the brightness temperature of $2245 \mathrm{~K}$. All $\mathrm{cm}$ datapoints for $\mathrm{N} 1$ can be well-fitted with a power law of slope 1.36. The shallow slope and the high brightness temperature indicate that the measured $7 \mathrm{~mm}$ flux density in the $\mathrm{A}+\mathrm{B}$ configuration data is not due to dust emission. Power-law spectra with slopes between 1-2 are often found for massive protostellar candidates (e.g. Hofner et al. 1996; Beuther et al. 2004) and can be explained by free-free emission from ionized gas where a density gradient causes partially optically-thick conditions (Franco et al. 2000; Keto 2003; see also Ignace \& Churchwell 2004).

Having thus established the nature of the radio continuum emission in our high resolution data from N1 as ionized gas, we can ask what causes the ionization. The answer to this question will depend strongly on the position of the protostar, which as discussed earlier is still unknown.

First, we point out that the direction of the proper motion of the $\mathrm{H}_{2} \mathrm{O}$ masers excludes a position of the protostar between $\mathrm{N} 1$ and N2; the cm continuum jet from IRAS20126+4104 is clearly one-sided. Consider then the possibility of direct photoionization by the protostar. If we assume that the protostar is located at the position of N1, we can calculate the necessary ionizing flux to produce the observed continuum emission. Assuming optically thin conditions and a temperature of $10^{4} \mathrm{~K}$ for the ionized gas, we follow the method outlined in Kurtz, Churchwell \& Wood (1994) or Schraml \& Mezger (1969), to calculate the Lyman continuum flux $N_{\mathrm{c}}$ necessary for maintaining the observed $7 \mathrm{~mm}$ continuum flux density of $1.38 \mathrm{mJy}$ and find $N_{\mathrm{c}} \sim 4.4 \times 10^{44} \mathrm{~s}^{-1}$. This flux of ionizing photons would correspond to a ZAMS star of mass of about $7 M_{\odot}$, similar to the mass deduced from the kinematic studies of $\mathrm{C} 05$. However, this is clearly a lower limit, due to dust absorption, and optical depth effects in conjunction with the unknown distribution of the ionized medium. Also, this scenario does not explain the existence of N2, as this would require a larger Lyman continuum flux, inconsistent with a $7 M_{\odot}$ star. In conclusion, these arguments appear to exclude direct photoionization by a star located in N1.

Alternatively, we can assume that the position of the massive protostar is located near the centroid of the $\mathrm{OH}$ maser emission and located within the dark lane as suggested by Edris et al. (2005) and Sridharan et al. (2005), at $\alpha(J 2000)=$ $20^{\mathrm{h}} 14^{\mathrm{m}} 26^{\mathrm{s}} 05, \delta(J 2000)=41^{\circ} 13^{\prime} 32^{\prime \prime}$. 45 (see Figs. 1 and 2 ). In this scenario the radio continuum emission consisting of the two sources N1 and N2 represent a one-sided jet driven by the central object. The cause of the ionization of $\mathrm{N} 1$ and $\mathrm{N} 2$ could then be either direct photo-ionization from the central object or ionization from UV photons from the cooling region of a shock caused by mass outflow from the central object, interacting with the surrounding molecular medium (e.g. Curiel et al. 1989).

In the former case we again calculate a lower limit on the Lyman continuum flux of the central object assuming opticallythin free-free emission from N1. Taking into account the solid angle subtended by $\mathrm{N} 1$ as seen from the position of the central protostar of $\Omega / 4 \pi=0.01$, a Lyman continuum flux of $N_{\mathrm{c}}>$ $4.4 \times 10^{46} \mathrm{~s}^{-1}$ is needed to produce the observed flux density. If this were supplied by a main-sequence star, the corresponding bolometric luminosity $\left(\sim 10^{4} L_{\odot}\right)$ would be very close to the total luminosity of IRAS $20126+4104$. However, this seems unlikely as a fraction of the luminosity must be contributed by other (lower-mass) stars in the cluster. Furthermore, no radio continuum emission was detected at the hypothetical position of the proto-star. This is consistent with the large accretion rate found by C99, C05, which can essentially keep the ionization front near the stellar surface (Walmsley 1995) even for ZAMS stars. However, if N1 were ionized directly by radiation of the protostar, paths must exist along which the ionizing photons can reach $\mathrm{N} 1$, and we should observe some ionization along the boundary of the accretion flow, which is not observed. We thus consider this scenario for ionization of $\mathrm{N} 1$ as unlikely.

Based on the above considerations we consider it most likely that the ionization of N1 (and N2) are caused by shocks from outflowing material. The bow-shock-like morphology of N2, 
although weak, is suggestive of this scenario. Also $\mathrm{H}_{2} \mathrm{O}$ maser emission is associated with both $\mathrm{N} 1$ and $\mathrm{N} 2$, and at least the masers near $\mathrm{N} 1$ are moving outward with velocities between $30-100 \mathrm{~km} \mathrm{~s}^{-1}$. Although it is difficult to relate the observed level of ionization to the physical parameters of the shock, the theory outlined by Curiel et al. (1989) can be used for an order-of-magnitude check. Using their Eq. (8) and assuming an isotropic mass loss rate of $\dot{M}_{\text {out }}=10^{-2} M_{\odot} \mathrm{yr}^{-1}$ (C99), a temperature of $10^{4} \mathrm{~K}$ for the ionized gas, and a outflow velocity of $100 \mathrm{~km} \mathrm{~s}^{-1}$, the predicted flux density is about $1 \mathrm{mJy}$, close to what is measured.

It is also worth noting that, if the above interpretation is correct, then IRAS 20126+4104 must be a massive protostar, namely a massive star still in the main accretion phase. In fact, this is the only way to prevent formation of an HII region around a B0.5 star. Ionization in the plane of the disk is prevented by the steep density gradient due to the accreting material, while along the disk axis the HII region could be confined by the jet itself, as predicted in the model by Tan \& McKee (2003).

\subsection{The southern jet}

To extract physical parameters from our $3.6 \mathrm{~cm}$ image of the southern jet, we assume that the emission at this wavelength is optically-thin free-free emission. This assumption is consistent with our data as the brightness temperature averaged over the source is only $25 \mathrm{~K}$, and our detection limits at 1.3 and $0.7 \mathrm{~cm}$ at the peak position imply spectral indices less than 0.8 between $3.6 \mathrm{~cm}$ and these wavelengths. Most jets from highly embedded sources are thermal, with the exception of $\mathrm{W} 3\left(\mathrm{H}_{2} \mathrm{O}\right)$ (Reid et al. 1995) whose radio jet has a spectral index of -0.6 . Assuming a similar spectral index for IRAS $20126+4104$ S, we should have detected this source at $20 \mathrm{~cm}$; however, our non-detection at this wavelength constrains the spectral index between 3.6 and $20 \mathrm{~cm}$ to larger than -0.2 , i.e. in the range expected for thermal freefree emission.

A possible model for the $3.6 \mathrm{~cm}$ continuum emission is photoionization by stellar UV radiation that is confined to the solid angle of the jet by a non-symmetric circumstellar mass distribution that could be due to the presence of an accretion disk. From our non-detection of the $7 \mathrm{~mm}$ continuum, we can only place an upper limit on the presence of matter around IRAS $20126+4104 \mathrm{~S}$ of about $1.8 M_{\odot}$, which due to the low value of the dust emissivity at this wavelength is not a strong constraint. The non-detection of IRAS $20126+4104 \mathrm{~S}$ in the $1.3 \mathrm{~mm}$ observations of C99 (angular resolution of $\sim 0.7$ ) implies an upper limit on the dust mass of $0.4 M_{\odot}$ at the position of IRAS $20126+4104 \mathrm{~S}$. The measured dimensions of the ionized jet imply that collimation occurred within a distance of $500 \mathrm{AU}$ from the central source with a collimation ratio (ratio of length/width of jet) of $\sim 5$ and a solid angle, $\Omega$, of the emission cone satisfying $\Omega / 4 \pi=0.02$. Assuming an electron temperature $T_{\mathrm{e}}=10^{4} \mathrm{~K}$, we derive an average optical depth $\tau_{3.6 \mathrm{~cm}}=0.0025$, corresponding to an emission measure of about $6.8 \times 10^{5} \mathrm{~cm}^{-6} \mathrm{pc}$. Asssuming that the extent of the ionized region along the line of sight is equal to its observed minor axis of $0 !^{\prime} 15$, we estimate a mean electron density of $n_{\mathrm{e}}=2.4 \times 10^{4} \mathrm{~cm}^{-3}$. Neglecting the absorption of ionizing photons by dust, the measured $3.6 \mathrm{~cm}$ flux of $0.15 \mathrm{mJy}$ then corresponds to a total Lyman continuum flux of $1.8 \times 10^{45} \mathrm{~s}^{-1}$. If this is supplied by a ZAMS star, then the corresponding spectral type is B1, which in turn has a bolometric luminosity of about $5 \times 10^{3} L_{\odot}$, or $50 \%$ of the luminosity in the region.
Alternatively, the $3.6 \mathrm{~cm}$ emission could be caused by shock ionization, as discussed above for the northern sources (see also Hofner et al. 1999). Our data do not allow a clear distinction between these two scenarios, although we consider shock ionization of IRAS $20126+4104$ S more likely for the following reason. Although of similar spectral types (B1 and B0.5), sources S and $\mathrm{N} 1$ are remarkably different in almost every other aspect: $\mathrm{S}$ is detected only in the $3.6 \mathrm{~cm}$ continuum emission, whereas N1 is seen in a large variety of tracers, all of which demonstrate that $\mathrm{N} 1$ is associated with a disk at least 10 times more massive than the (putative) disk focusing the jet in S. This suggests that the disk in $\mathrm{S}$ has been almost completely dissipated, which conflicts with the idea that the ionizing flux is focused by the disk. In this situation, one should see a more spherical HII region rather than an elongated, jet-like structure.

The orientation of the IRAS $20126+4104 \mathrm{~S}$ jet is very close to the orientation of the molecular flow observed on a larger scale e.g. in $\mathrm{HCO}^{+}$(C97). Thus the question arises whether IRAS 20126+4104 S contributes to the large-scale molecular flow. If one computes the momentum rate of the ionized jet in IRAS 20126+4104 S using either the theory of Reynolds (1986) or Curiel et al. (1989), in both cases one arrives at momentum rates that are several orders of magnitude lower than deduced from the molecular line observations that trace the bipolar flow from the region (see Hofner et al. 1999). This discrepancy also appears for the northern sources and in fact is commonly observed in flow/jet sources and could be due to a neutral jet component. Thus, it is not possible to quantify the contribution of IRAS $20126+4104 \mathrm{~S}$ to the bipolar molecular flow based on the radio continuum data. Nonetheless, it is worth noting that the dominant contribution to the bipolar outflow very likely comes from the northern source. This is demonstrated by a large number of observations of the kinematics and structure of the flow from parsec-scales (Shepherd et al. 2000) to a few $100 \mathrm{AU}$ (Moscadelli et al. 2000, 2005), which show that source N1 lies near the origin of the flow. Therefore, although a contribution from IRAS $20126+4104 \mathrm{~S}$ cannot be excluded, this is unlikely to be the dominant contribution to the overall mass and energy of the flow.

\subsection{Spectral energy distribution}

In Fig. 5 we show the spectral energy distribution (SED) of IRAS 20126+4104 from radio wavelengths to the mid-infrared region. There are no other signposts of massive star formation in the vicinity of the IRAS $20126+4104$ core, and thus the SED shown in Fig. 5 is dominated by the energetics of the central objects.

The work presented here adds information about the smallest scales $(\approx 100 \mathrm{AU})$, where the dominant emission is from ionized gas. Our data are consistent with the detailed picture of a disk/jet system, which was elaborated in a series of papers by several authors (C97; C99; C05; Hofner et al. 1999; Zhang et al. 1998; Edris et al. 2005). We used a simple radiative transfer model (see Olmi et al. 2003) including both a spherical halo and a cylindrical accretion disk to model the overall SED in IRAS 20126+4104. Since the above studies indicate that the bipolar flow in IRAS 20126+4104 within a few arcseconds from the star is located very near the plane of the sky, we assumed an edge-on orientation for the accretion disk in our model. Also note that the dark lane reported by Sridharan et al. (2005) is consistent with this assumption. Following Kramer et al. (1998), we adopted a total (dust+gas) absorption coefficient $\kappa(v)=0.005 \mathrm{~cm}^{-2} \mathrm{~g}^{-1}(v / 231 \mathrm{GHz})^{\beta}$, with $\beta=1.2$. The 


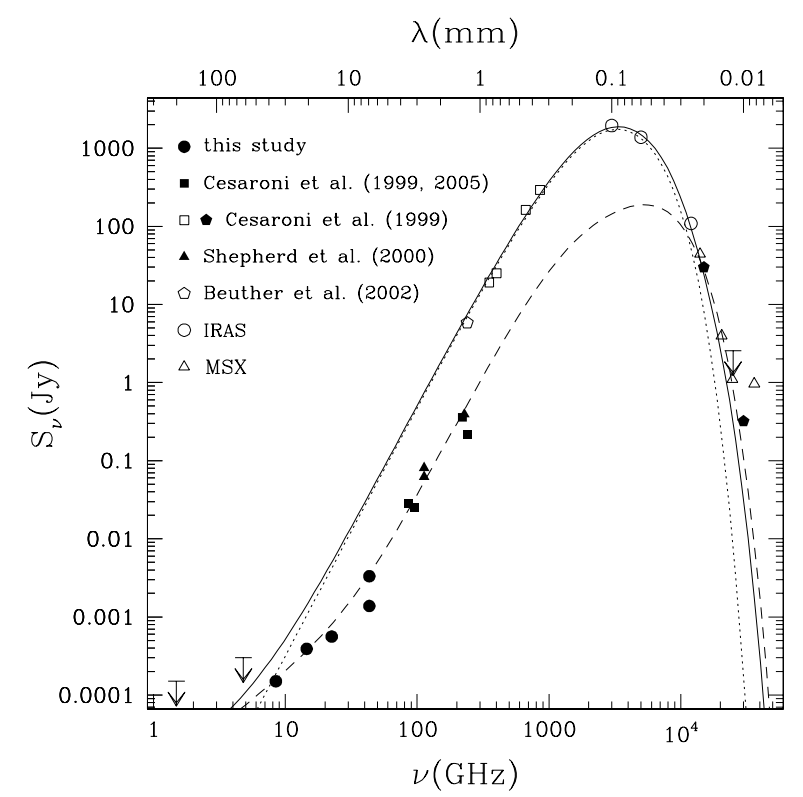

Fig. 5. Spectral energy distribution of the IRAS 20126+4104 core region. The radio/mm section of this figure is identical to Fig. 4. Filled data points indicate data with high angular resolution (arcsecond or smaller), and open data points indicate data of low angular resolution (tens of arcseconds). The dashed line indicates the emission from the accretion disk plus the power law describing the radio emission. The dotted line shows the emission from the spherical halo, and the solid line describes the total spectrum taking into account absorption of disk emission by the halo material at IR wavelengths.

value of $\beta$ was obtained from the mm-submm part of the SED. The model of the spherical envelope shown in Fig. 5 has an outer radius of $0.54 \mathrm{pc}$ and an inner radius of $0.0041 \mathrm{pc}$ (850 AU) where it connects to the accretion disk. The temperature follows a power law, $\mathrm{T} \propto r^{q}$, where $q=-2 /(4+\beta)=-0.38$ (e.g. Doty \& Leung 1994), with $T=25 \mathrm{~K}$ at the outer radius. We adopt an infall law for the run of density in the envelope, i.e. $n \propto r^{-3 / 2}$. The total mass and luminosity for the envelope are $402 M_{\odot}$ and $8100 L_{\odot}$. The accretion disk is modeled as an edge-on cylinder of height $640 \mathrm{AU}$, an inner radius of $34 \mathrm{AU}$, and an outer radius of $850 \mathrm{AU}$. The density is assumed to be constant $\left(10^{9} \mathrm{~cm}^{-3}\right)$ and we use the typical temperature law $T \propto r^{-0.75}$ for disks (e.g., Pringle 1981). The total mass and luminosity of the disk are $6 M_{\odot}$ and $1540 L_{\odot}$.

Inspection of Fig. 5 shows that the envelope-disk model described above provides a satisfactory description of the SED of IRAS 20126+4104. The interferometric data in the $\mathrm{cm} / \mathrm{mm}$ wavelength range are not sensitive to the extended envelope, and hence fit only the dashed line that describes the radio emission from the ionized gas and the dust emission from the disk. In contrast, the envelope is detected in the submm/FIR wavelength region with single-dish telescopes. In the mid-IR region the disk dominates the emission; however, absorption from the envelope reduces its flux, so that the total emerging radiation in this wavelength range falls below that of the accretion disk alone.

In conclusion, although our envelope/disk model for IRAS $20126+4104$ is consistent with the observed SED, more sophisticated models (e.g. Indebetouw et al. 2006) should be constructed, taking into account the effects of envelope geometry, clumpiness, grain sizes, bipolar cavities, disk geometry (e.g. flaring, vertical extent), dust destruction radius, etc. However, our expectation is that the SED should be mostly affected in the near- to mid-IR regions of the spectrum, but not the flux at longer wavelengths $(\lambda>100 \mu \mathrm{m})$, which constrains the total mass of the envelope and disk. Therefore, these values should not change much when the effects mentioned above are taken into account. High quality data at $\lambda<100 \mu \mathrm{m}$ will be necessary for a more detailed radiative transport modeling effort; and when these become available, we expect that detailed information about the accretion disk geometry and composition can be extracted from future modeling.

\section{Summary}

We have performed observations with the VLA in the A and B configurations at wavelengths of $20,6,3.6,1.3$, and $0.7 \mathrm{~cm}$ toward the IRAS $20126+4104$ core region. At $3.6 \mathrm{~cm}$ we detected three sources. The two northern sources are best explained as a manifestation of shocked gas caused by the jet driven by the central object. Our radio data cannot conclusively determine the position of the central object, but they are consistent with the central protostar being located about $0{ }^{\prime} 3$ to the SE of the main $3.6 \mathrm{~cm}$ continuum source as suggested by Sridharan et al. (2005). The southern continuum source has the morphology of an ionized jet, and its $3.6 \mathrm{~cm}$ emission is consistent with optically thin free-free radiation.

We have computed a simple radiative transfer model consisting of an edge-on accretion disk surrounded by a spherical halo to describe the SED for the IRAS 20126+4104 region. We take into account the different angular resolutions of the data used to fit the SED and conclude that the data are consistent with our model.

We also report the detection of a highly variable radio source located about $5^{\prime \prime}$ to the SE of the IRAS 20126+4104 core. This source was detected in 6 of the 12 datasets presented in this paper. The characteristics of the $\mathrm{cm}$-variability from this source are consistent with gyrosynchotron emission from a pre-main sequence low-mass (T Tauri) star.

Acknowledgements. The authors thank G. Fuller and J. M. Torrelles for helpful discussions. P. H. acknowledges support from NSF grant AST-0454665. This research made use of the NASA/IPAC Extragalactic Database (NED), which is operated by the Jet Propulsion Laboratory, California Institute of Technology, under contract with the National Aeronautics and Space Administration. We thank the anonymous referee for comments that improved the presentation of this paper.

\section{References}

Ayala, S., Curiel, S., Raga, A. C., Noriega-Crespo, A., \& Salas, L. 1998, A\&A, 332,1055

Beuther, H., Zhang, Q., Greenhill, L. J., et al. 2004, ApJ, 616, 31

Cesaroni, R., Felli, M., Testi, L., Walmsley, C. M., \& Olmi, L. 1997, A\&A, 325, 725

Cesaroni, R., Felli, M., Jenness, Neri, R., et al. 1999, A\&A, 345, 949

Cesaroni, R., Neri, R., Olmi, L., et al. 2005, A\&A, 434, 1039

Coffey, D., Bacciotti, F., Woitas, J., Ray, T. P., \& Eislöffel, J. 2004, ApJ, 604, 758

Curiel, S., Rodríguez, L.F., Canto, J., et al. 1989, Astrophys. Lett. Comm., 27, 299

Doty, S. D., \& Leung, C. M. 1994, ApJ, 424, 729

Edris, K. A., Fuller, G. A., Cohen, R. J., \& Etoka, S. 2005, A\&A, 434, 213

Felli, M., Taylor, G. B., Catarzi, M., Churchwell, E., \& Kurtz, S. 1993, A\&ASS, 101,127

Franco, J., Kurtz, S., Hofner, P., et al. 2000, ApJ, 542, L143

Garmire, G., Feigelson, E. D., Broos, P., et al. 2000, AJ, 120, 1426

Gómez, Y., Rodríguez, L. F., \& Garay, G. 2002, ApJ, 571, 901

Hofner, P., Kurtz, S., Churchwell, E., Walmsley, C. M., \& Cesaroni, R. 1996, ApJ, 460, 359

Hofner, P., Cesaroni, R., Rodríguez, L. F., \& Martí, J. 1999, A\&A, 345, L43

Ignace, R., \& Churchwell, E. 2004, ApJ, 610, 351

Keto, E. 2003, ApJ, 599, 1196 
Kramer, C., Alves, J. \& Lada, C. 1998, A\&A, 329, L33

Kurtz, S., Churchwell, E., \& Wood, D. O. S. 1994, ApJS, 91, 659

Moscadelli, L., Cesaroni, R., \& Rioja, M. J. 2000, A\&A, 360, 663

Moscadelli, L., Cesaroni, R., \& Rioja, M. J. 2005, A\&A, 438, 889

Olmi, L., Cesaroni, R., Hofner, P., et al. 2003, A\&A, 407, 225

Pringle, J. E. 1981, ARA\&A, 19, 137

Pudritz, R. E., \& Banerjee, R. 2005, IAU Symp. 227, ed. R. Cesaroni, M. Felli, E. Churchwell, \& M. Walmsley (Cambridge: Cambridge University Press), 163

Reid, M. J., Argon, A. L., Masson, C. R., Menten, K. M., \& Moran, J. M. 1995, ApJ, 443, 238

Reynolds, S. P. 1986, ApJ, 304, 713

Schraml, \& Mezger 1969, ApJ, 156, 269
Shepherd, D. S., Yu, K. C., Bally, J., \& Testi, L. 2000, ApJ, 535, 833 Sridharan, T. K., Williams, S. J., \& Fuller, G. A. 2005, ApJ, 631, L73

Tan, J. A., \& McKee, C. F. 2003, in Star Formation at High Angular Resolution, IAU Symp., 221

Tofani, G., Felli, M., Taylor, G. B., \& Hunter, T. R. 1995, A\&AS, 112, 299

Trinidad, M. A., Curiel, S., Migenes, V., et al. 2005, AJ, 130, 2206

Walmsley, M. 1995, Rev. Mex. Astron. Astrofis. Ser. Conf., 1, 137

Windhorst, R. A., Fomalont, E. B., Partridge, R. B., \& Lowenthal, J. D. 1993, ApJ, 405, 498

Woitas, J., Bacciotti, F., Ray, T. P., et al. 2005, A\&A, 432, 149

Zapata, L. A., Rodríguez, L. F., \& Kurtz, S. E. 2004, ApJ, 127, 2252

Zhang, Q., Hunter, T. R., \& Sridharan, T. K. 1998, ApJ, 505, L151 